\title{
Meeting Report and Special Issue Preface: International Digestive Endoscopy Network (IDEN) 2016
}

\author{
Sang Kil Lee ${ }^{1}$ and Jong Kyun Lee ${ }^{2}$ \\ ${ }^{1}$ Department of Internal Medicine, Severance Hospital, Yonsei University College of Medicine, Seoul, ${ }^{2}$ Department of Medicine, Samsung \\ Medical Center, Sungkyunkwan University School of Medicine, Seoul, Korea
}

The Korean Society of Gastrointestinal Endoscopy (KSGE) has been hosting the International Digestive Endoscopy Network (IDEN) since 2011. IDEN 2016 was held at the Grand Hilton Hotel, Seoul, Korea, from June 24 to 26, 2016. The KSGE collaborated with the American Society for Gastrointestinal Endoscopy (ASGE) in hosting the KSGE-ASGE joint sessions in the conference.

At IDEN 2016, many physicians and researchers from all over the world participated to present and discuss the latest advances in the field of upper gastrointestinal (GI), lower GI, and pancreatobiliary endoscopies. The scientific programs included live demonstrations of cutting-edge practice and skills in GI endoscopy. A total of 82 speakers were invited from overseas and Korea. The program consisted of more than 27 sessions with live demonstrations, lectures, case-based discussions, oral presentations by Asian Young Endoscopist Award
(AYEA) 2016 participants and 154 poster presentations. IDEN 2016 also included special programs such as the AYEA and Young Endoscopist Forum for young endoscopists from Asian countries. AYEA participants received clinical training in GI endoscopy at selected endoscopy training centers for 2 weeks before the IDEN in Korea. They acquired valuable learning in these programs. The number of participants was over 776, reflecting the great interest of the medical community from all over the world.

The seminars have contributed much to the spread of knowledge about GI endoscopy. Indeed, the IDEN has become among the most attractive international conferences held in Korea. Next year, IDEN will be held in June 2017.

Conflicts of Interest

The authors have no financial conflicts of interest.
Received: September 16, 2016

Correspondence: Jong Kyun Lee

Department of Medicine, Samsung Medical Center, Sungkyunkwan University School of Medicine, 81 Irwon-ro, Gangnam-gu, Seoul 06351, Korea

Tel: +82-2-3410-3410, Fax: +82-2-3410-6983, E-mail: jongkyunlee@gmail.com

(cc) This is an Open Access article distributed under the terms of the Creative Commons Attribution Non-Commercial License (http://creativecommons.org/ licenses/by-nc/3.0) which permits unrestricted non-commercial use, distribution, and reproduction in any medium, provided the original work is properly cited. 